\title{
EDAD, GÉNERO Y ESTATUS PROFESIONAL DE LOS INVESTIGADORES COMO INDICADORES DE LA PERCEPCIÓN DE TWITTER EN LA DIFUSIÓN DE LA CIENCIA
}

\author{
Dr. () Francisco Javier Alonso Flores \\ Universidad Carlos III de Madrid, Madrid, España \\ fjalonso@bib.uczm.es \\ ORCID iD: https://orcid.org/0000-0003-4696-4684 \\ Dra. Carolina Moreno Castro \\ Universitat de València, Valencia, España \\ Carolina.Moreno@uv.es \\ ORCID iD: https://orcid.org/0000-0001-7453-4257 \\ Dr. Antonio Eleazar Serrano \\ Universidad Carlos III de Madrid, Madrid, España \\ aeserran@bib.uczm.es \\ ORCID iD: https://orcid.org/0000-0003-1261-386X
}

Recibido el 31 de octubre de 2018

Aceptado el 2 de enero de 2019

\section{Resumen}

El objetivo de este estudio es evaluar el grado de satisfacción e interés que presenta el personal de investigación de las universidades españolas por las redes sociales como Twitter. Para ello, se han examinado los datos obtenidos mediante una encuesta online a unos 600 investigadores proactivos de la divulgación científica de 20 universidades españolas (2016). Para el análisis de los datos, se utilizó el test de independencia de Kruskal-Wallis. Según los resultados, dos de cada tres (65.4\%) investigadores percibieron beneficios después de haber realizado la comunicación de sus resultados de investigación y una gran mayoría $(84.7 \%)$ no percibieron ningún perjuicio. En relación con las redes sociales, tres de cada cuatro investigadores (74.6\%) opina que son un instrumento importante para mejorar la comunicación científica, aunque menos de la mitad (un 41.4\%) tienen un perfil abierto activo en redes sociales como Twitter. Además, las investigadoras perciben las redes sociales como un medio más importante que los investigadores. Y quienes tienen perfil activo en redes sociales, las valoran mejor.

Palabras clave: Redes Sociales, Noticias Científicas, Comunicación Institucional, Universidades, Comunicación Científica, Divulgación Científica. 


\title{
RESEARCHERS' AGE, GENDER AND PROFESSIONAL STATUS AS INDICATORS OF THE TWITTER PERCEPTION IN SCIENCE DISSEMINATION
}

\begin{abstract}
The objective of this study is to evaluate the level of satisfaction and interest that researchers from Spanish universities present through social networks like Twitter. To do so, we examined the data obtained through an online survey from over 600 researchers proactive in science dissemination from 20 Spanish universities (2016). In regard to the data analysis, Kruskal-Wallis independence test was used. According to the results, two out of three researchers (65.4\%) perceived benefits after the science communication campaign and a large majority (84.7\%) did not perceive any damage. In relation to social networks, three out of four researchers (74.6\%) think that it is an important instrument to improve scientific communication, although less than half (41.4\%) have an active open profile in social networks such as Twitter. In addition, female researchers perceive social networks to be more important than male researchers consider them to be, and those who have an active profile in social media networks value them even greater.
\end{abstract}

Keywords: Social Networks, Science News, Institutional Communication, Universities, Science Communication, Science Popularization.

\section{Cómo citar este artículo:}

Alonso, F., Moreno, C., Eleazar, A. (2019). "Edad, Género Y Estatus Profesional De Los Investigadores Como Indicadores De La Percepción De Twitter En La Difusión De La Ciencia", en Perspectivas de la Comunicación, Vol. 12, № 1. pp. 157-184. 
Introducción

A

ctualmente los investigadores que quieren realizar divulgación científica se encuentran en un proceso de adaptación al entorno digital. El tránsito está siendo desigual, en función de la edad, la experiencia en la investigación, el género o de su propia predisposición. Hasta ahora, sus artículos y sus debates académicos se hallaban en bases de datos encriptadas a las que solo se podía acceder mediante pago. Sin embargo, desde hace una década en España, ha habido intentos por parte de las universidades públicas y privadas de visibilizar a través de los medios de comunicación convencionales $y$, sobre todo, de las redes sociales la producción científica de sus investigadores (Parejo, Martín y Vivas, 2017). Este momento ha coincidido con el periodo en el que todas las encuestas indican que una inmensa mayoría de las personas utilizan las redes sociales y los motores de búsqueda para encontrar información de interés, y donde más de la mitad de la población indica que Internet es su principal fuente informativa (Brossard y Scheufele, 2013: 40; FECYT, 2018). Por ello, ha surgido una nueva urgencia para que los científicos presten atención a estas tendencias.

La comunicación pública de la ciencia está comenzando a formar parte del conjunto de conocimientos o habilidades necesarias para ser relevante como científico (Chapman et al, 2015; Peoples et al, 2016). Comunicar y divulgar la ciencia es un paso más en el proceso de investigación, que no finaliza en el artículo científico, sino en la transmisión al gran público de los resultados de Investigación, Desarrollo e Innovación (I+D+i). De hecho, hay un número creciente de trabajos académicos que tratan de proporcionar consejos prácticos sobre cómo abordar la comunicación científica desde diferentes perspectivas, como las redes sociales, los blogs, la eficiencia y originalidad en la comunicación del I+D+i, etc. (Bik y Goldstein, 2013; Dennen, 2014; Cooke et al, 2017; Pérez Rodríguez, González Pedrás \& Alonso Berrocal, 2018). E incluso existen otros trabajos académicos que pueden parecer pequeños manuales de redacción periodística adaptados al mundo de la popularización de la ciencia, con recomendaciones sobre cómo utilizar técnicas de storytelling, plantear el enfoque de los temas invirtiendo el orden clásico de los artículos científicos para empezar hablando de las conclusiones o cómo reconocer y adaptar el estilo para dirigirse específicamente a una audiencia determina (Babbit, 2018: 1735).

El desarrollo en los últimos años de las redes sociales digitales científicas (RSDC), como ResearchGate y Academia.edu, ha supuesto un nuevo punto de encuentro para los investigadores, ya que son plataformas ágiles para difundir resultados de las investigaciones. Sin embargo, según un trabajo relativamente reciente, que analiza la presencia del personal académico adscrito a setenta y siete universidades públicas y privadas españolas, se concluye que en estas dos RSDC todavía no se ha generalizado su utilización en España (González-Díaz, Iglesias-García, Codina, 2015: 640). A pesar del desarrollo que están experimentando en todo el mundo, el número de miembros españoles en estas redes es insuficiente y en el caso de algunas universidades resulta casi anecdótico, si se contempla como objetivo de la difusión del conocimiento. Otro estudio centrado en universidades de Galicia (España) apunta al bajo conocimiento y al uso eficiente de redes como ResearchGate, dado que menos de un $33 \%$ de los profesores las utilizan, al menos una vez al mes, para difundir publicaciones o para conseguir más citas y reputación (Campos-Freire, Rúas-Araujo, 2016: 431). Por ello, resulta tan extremadamente 
interesante estudiar la utilización que los investigadores hacen de otras redes sociales más generalistas.

Algunos científicos promueven su trabajo por su cuenta a través de blogs o redes sociales como Twitter y Facebook, pero en pocas ocasiones pueden conseguir las grandes audiencias de los principales medios de comunicación. Por eso, la revista Nature (Kwok, 2018: 271) recomienda que contacten con las oficinas de prensa de sus centros de investigación. Por su parte, las instituciones públicas desean tener cada vez más visibilidad y transparencia de sus actividades ante la ciudadanía porque estos dos indicadores les otorgan cierta imagen de honestidad (Moreno, Molina y Corcoy, 2013: 21). Además, en el caso de las universidades, la visibilidad de su I+D+i puede mejorar su posición en los rankings internacionales al mejorar su reputación (Johnes, 2018; Pérez-Esparrells \& LópezGarcía, 2018).

En las universidades públicas españolas este trabajo se realiza básicamente a través de los gabinetes de comunicación institucional y de las Unidades de Cultura Científica y de Innovación (a partir de ahora, UCC+i). La Red de UCC+i dio sus primeros pasos con motivo de la publicación de la Convocatoria de Ayudas para la realización de actividades de difusión y divulgación científica y tecnológica del Año de la Ciencia 2007, promovido por la Fundación Española para la Ciencia y la Tecnología (FECYT), que incentivó la creación y el fortalecimiento de este tipo de unidades en universidades y centros de investigación en España (FECYT, 2012: 7). Desde entonces, las diferentes UCC+i que se han ido creando en las universidades españolas han enfocado su actividad hacia ámbitos diferentes, tales como: la organización de actividades de divulgación (ferias de la ciencia, exposiciones, talleres, concursos, etc.), las acciones formativas dirigidas a la comunidad investigadora y educativa, la comunicación científica y las relaciones con los medios de comunicación (FECYT, 2016). Dichas estructuras surgieron para responder a la necesidad de difundir lo que ocurría en materia científica y tecnológica dentro de las instituciones, teniendo en cuenta que entre su público interno se encuentran los investigadores, que son al mismo tiempo generadores y fuente para divulgar la información científica (Roca Marín, 2017). En la actualidad, estas UCC+i están presentes en casi el $40 \%$ de las universidades españolas y una treintena de ellas contaban con este servicio específico de comunicación dedicado a la labor de acercar la ciencia a la ciudadanía en sus diferentes formatos (Parejo, Martín-Pena y Pinto-Zúñiga, 2016: 532).

Tal y como recogen Alonso-Flores, Eleazar Serrano-López y Moreno-Castro (2018), las universidades españolas han tardado décadas en integrar los gabinetes de prensa a su organigrama de servicios y, por ende, en ser conscientes de la importancia que tenían estos departamentos para sus instituciones. De hecho, no fue hasta la llegada de la democracia (años 70 y 80) cuando se comenzaron a impulsar los primeros servicios de prensa en los campus universitarios españoles, con el objetivo de lograr cierta proyección pública a través de los medios de comunicación, algo a lo que hasta la fecha no se había prestado atención (Moreno Castro, 2004; Paniagua Rojano, Gómez Calderón \& Fernández Sande, 2012). No obstante, los gabinetes de comunicación en las universidades se incrementaron durante la década de los go sobre todo, con la implantación de nuevas universidades privadas, momento en el que se comenzaron a diseñar nuevas técnicas de mercadotecnia con las que difundir la imagen de la institución (Parejo Cuéllar, 2016). Históricamente, las funciones de estos gabinetes de prensa han estado focalizadas en las actividades que estuvieran planificadas en la agenda del rector; en las peticiones de entrevistas por parte de los medios de comunicación; en la difusión de notas de prensa de carácter totalmente 
institucional y, finalmente, en la emisión de algunas notas de prensa con los resultados de I+D+i de algún grupo de investigación.

Diversos estudios han señalado que los medios de comunicación y las universidades públicas son dos pilares importantes a la hora de incrementar el nivel de cultura científica entre los jóvenes españoles, mediante el uso de Internet y la Web 2.0 (Olvera-Lobo y López-Pérez, 2014). En el caso de las universidades españolas, los resultados demuestran el esfuerzo que están haciendo para conectar la ciencia con estas herramientas: el 72.9\% de las universidades difunden noticias científicas frecuentemente y casi un tercio tienen un perfil en Facebook y Twitter, que alimentan con noticias sobre la producción científica de sus investigadores. Un estudio reciente (González-Pedraz, Pérez-Rodríguez, CamposDominguez, Quintanilla, Fisac, 2018:169) sugiere que las UCC+i están favoreciendo la visibilidad de la ciencia española y que los medios digitales con escasos recursos dependen informativamente de ellas. Sin embargo, el papel de la ciencia española sigue siendo irrelevante en los periódicos online. Esto supone una constante desde hace décadas. El fenómeno no es solo español, sino internacional (Garimella y Xiao, 2017), ya que es muy poco probable que una investigación llegue a ser mediática y las que llegan a serlo tienen poca cabida en los medios de comunicación.

A pesar de que los medios tradicionales (diarios y revistas en papel, radio, televisión, y sus respectivas versiones online) están en decadencia y el número de usuarios es cada vez menor, habiéndose producido una gran crisis en el sistema actual de medios, la mayoría de los científicos aún los siguen considerando como los principales canales de comunicación con el público (Allgaier et al. a), 2013). Esto supone un conflicto con el interés público por los "nuevos medios de comunicación", como los blogs y redes sociales. Es como si los científicos estuvieran interesados por unos medios de comunicación y la sociedad estuviera interesada por otros canales de comunicación. Sin embargo, según otro estudio de los mismos autores (Allgaier et al. b), 2013), basado en entrevistas con neurocientíficos de Alemania y EEUU, la mayoría de investigadores considera la comunicación con el público una obligación moral y una necesidad estratégica; pero otros consideran que les distrae de su verdadero trabajo (Hall, 2014). Este autor incluso ha propuesto un índice para demostrar que quienes se dedican a alimentar perfiles públicos de redes sociales, alimentándolas con la producción científica, publican menos papers que quienes solo se dedican a la investigación. Según este autor, "si bien los medios sociales son una herramienta valiosa para la difusión y el intercambio de ideas, existe el peligro de que esta forma de comunicación gane un valor demasiado alto y de que perdamos de vista las métricas críticas de valor científico, como los índices de citas. Para ayudar a cuantificar esto, propongo el 'Índice de Kardashian', una medida de discrepancia entre el perfil de los medios sociales de un científico y el registro de publicación basado en la comparación directa de números de citas y seguidores de Twitter".

Sin embargo, otros estudios apuntan justo en la dirección contraria. Según un trabajo de la Universidad de Wisconsin-Madison (Liang et al., 2014: 772), los científicos pueden incrementar las citaciones de sus trabajos académicos participando en discusiones científicas en redes sociales como Twitter. "Este estudio - según sus autores - proporciona la primera evidencia empírica exhaustiva de que las actividades de diseminación, como las interacciones con los reporteros y el ser mencionado en Twitter, pueden ayudar a la carrera del investigador mediante la promoción de su impacto científico". Otro estudio más reciente (Lamb, Gilbert y Ford, 2018) en el campo de la Ecología y la Conservación, señala que los investigadores pueden incrementar la exposición de su investigación a través de las 
redes sociales y, simultáneamente, mejorar su desempeño académico bajo las medidas tradicionales de la actividad investigadora.

Al igual que ya está ocurriendo en el campo de la comunicación política, que trata de adaptarse a las redes sociales como una nueva realidad social de los ciudadanos (ChavesMontero et al., 2017: 79), los investigadores que quieran conectar con la sociedad podrían tomar nota de las estrategias que se consideran exitosas en el ámbito político, como generar contenidos de calidad, con ideas propias que puedan generar expectación. Otros investigadores van más allá y, además del potencial de Twitter como herramienta para dar a conocer las publicaciones, hablan de la red social como un escenario informal para la revisión previa de manuscritos, de manera que podría convertirse en una valiosa contribución para las publicaciones científicas del siglo XXI (Darling et al, 2013). Por contra, otra corriente de opinión en el mundo de la investigación dice que los blogs y los tweets están destrozando artículos científicos en los días posteriores a su publicación, lo que deja a los investigadores sin saber cómo reaccionar (Mandavilli, 2011: 286).

Otros autores, proponen utilizar Twitter para construir una métrica innovadora para evaluar el impacto y la influencia de los trabajos académicos, con la gran ventaja de que se puede medir la aceptación de los resultados de investigación en muy pocos días y filtrar los hallazgos con el público en tiempo real (Eysenbach, 2011). Algunos estudios recientes comienzan a analizar los factores que pueden propiciar que unos contenidos científicos tengan más éxito que otros a nivel popular. Por ejemplo, en el ámbito del sector del ahorro de energía, los factores más significativos para el impacto en redes sociales de una investigación son la revista en que se publica el trabajo, la colaboración internacional y la clase de documentación (Filippo y Serrano López, 2018). En este contexto virtual y en el ámbito universitario, el trabajo en red resulta importante, según un trabajo de la Universidad Técnica de Delft (Países Bajos), que señala que las universidades pueden contribuir al buen desarrollo del campo de la comunicación científica, tanto en la teoría como en la práctica, si invierten en la construcción de colaboraciones y hacen uso del patrón de red que conecta a diversos actores, contextos y contenidos (Wehrmann y Van der Sanden, 2017:1). Porque lo que parece ya evidente es que, en el espacio de Twitter, en el que participa una amplia gama de actores, los investigadores deben jugar un papel importante en la creación directa de mensajes (Didegah, Mejlgaard y Sorensen, 2018: 960). Y por todo ello, también resulta necesario analizar su percepción sobre estas redes sociales.

\section{Objetivos y metodología}

El objetivo general de este estudio es conocer el interés por las redes sociales, como Twitter, que muestran los investigadores que participan activamente en la difusión de los resultados de sus trabajos a través de los gabinetes de comunicación institucionales y las UCCs. En concreto, se pretender analizar si las consideran útiles, si realmente las emplean para la difusión de sus investigaciones y si existe alguna diferencia por género, edad u otras características en relación a la percepción de las mismas.

Para poder conocer la opinión del personal investigador, se diseñó una encuesta, una técnica de investigación adecuada para estudio de actitudes, valores, creencias y motivos aplicable a casi todo el mundo y que permite estructurar y estandarizar fuertemente los datos (García Ferrando, 1992: 123). Así, aprovechando las ventajas que proporciona Internet en cuanto al análisis, difusión y flexibilidad en el acceso a la información de la encuesta (Ripoll Penadés, 2006: 41), se empleó una encuesta online computer-assisted web interview (CAWI). Uno de los problemas de este tipo de técnica es la baja tasa de 
respuestas, algo que se trató de paliar recurriendo al envío de correos electrónicos específicos a los investigadores, así como a través de un recordatorio adicional recordatorios adicionales. Además, el cuestionario se diseñó de tal manera que pudiera ser respondido de forma rápida (en un tiempo estimado de unos cinco minutos).

La encuesta constaba de casi una treintena de preguntas que se debían responder. Se han seleccionado las respuestas de aquellas cuestiones que resultaban adecuadas a los objetivos de esta investigación. De esta manera, había dos preguntas con respuestas dicotómicas (sí o no), para consultar a los investigadores sobre si habían participado en campañas de comunicación y si disponían de perfiles activos en redes sociales como Twitter. Otra pregunta se empleó para contextualizar el año en que se produjo su colaboración en la campaña de comunicación. Otra cuestión estaba relacionada con el grado de acuerdo (de 1 al 5) de los encuestados sobre si las redes sociales son un instrumento importante para mejorar la comunicación científica. Después se formulaba otra pregunta para realizar una valoración sobre si la campaña de comunicación había perjudicado o beneficiado al investigador (en una escala de 1 a 5 tipo Likert. En otras dos preguntas de marcador múltiple, se consultaba a los investigadores por posibles beneficios o perjuicios después de que su investigación se diera a conocer y apareciera en medios de comunicación. Además, se han tenido en cuenta 6 preguntas para la caracterización demográfica y recopilar información sobre la edad, género, años de experiencia en investigación, área científica, categoría profesional y procedencia institucional de los encuestados. En el Apéndice 1 aparecen todas estas preguntas y las posibles respuestas de forma completa.

Para la distribución de la encuesta, se solicitó la colaboración de las principales universidades públicas españolas que realizan acciones institucionales de comunicación del I+D+i. Por un lado, se contactó con las $35 \mathrm{UCC}+\mathrm{i}$ existentes en las universidades españolas en esa fecha a través de FECYT, la entidad del actual Ministerio de Ciencia, Innovación y Universidades que coordina esta red. Por otro lado, de forma complementaria, se contactó con los gabinetes de comunicación de las 38 universidades con una mayor tasa de visibilidad relativa en contenidos científicos en medios de comunicación, según un ranquin de las 67 instituciones científicas españolas por tasa de visibilidad relativa que apareció ese mismo año en una tesis doctoral de la Universidad de Salamanca (Pérez Rodríguez, 2016: 265). Después de informar de los términos de colaboración (señalando que los resultados se utilizarían para trabajos de investigación y que el tratamiento de los datos se haría de forma anonimizada), 20 universidades colaboraron distribuyendo el citado CAWI entre los investigadores que hubieran participado y colaborado en los últimos cinco años en noticias difundidas por estas entidades. En concreto, fueron las siguientes: la Universidad de Alcalá, de Alicante (UA), Autónoma de Barcelona (UAB), Autónoma de Madrid (UAM), de Barcelona (UB), Carlos III de Madrid (UC3M), Complutense de Madrid (UCM), de Córdoba (UCO), de Granada (UGR), de Jaén (UJA), Jaume I de Castellón (UII), de Málaga (UMA), Oviedo (UO), Politécnica de Madrid (UPM), Politécnica de Valencia (UPV), Rey Juan Carlos (URJC), Rovira y Virgili (URV), de Sevilla (US), de Valencia (UV) y de Zaragoza (UNIZAR).

Tabla 1. Investigadores contactados desde las universidades:

\begin{tabular}{|l|l|l|}
\hline Universidad & Investigadores & Respuestas obtenidas \\
\hline Alcalá (UAH) & 45 & 8 \\
\hline
\end{tabular}




\begin{tabular}{|c|c|c|}
\hline Alicante (UA) & 107 & 18 \\
\hline Autónoma de Barcelona (UAB) & 62 & 23 \\
\hline Autónoma de Madrid (UAM) & 200 & 31 \\
\hline Barcelona (UB) & 57 & 23 \\
\hline Carlos III de Madrid (UC3M) & 165 & 86 \\
\hline Complutense de Madrid (UCM) & 113 & 15 \\
\hline Córdoba (UCO) & 93 & 15 \\
\hline Granada (UGR) & 30 & 15 \\
\hline Jaén (UJA) & 65 & 11 \\
\hline Jaume I de Castellón (UII) & 152 & 22 \\
\hline Málaga (UMA) & 94 & 2 \\
\hline Oviedo (UO) & 153 & 21 \\
\hline Politécnica de Madrid (UPM) & 175 & 25 \\
\hline Politécnica de Valencia (UPV) & 175 & 63 \\
\hline Rey Juan Carlos (URJC) & 56 & 18 \\
\hline Rovira y Virgili (URV) & 44 & 7 \\
\hline Sevilla (US) & 678 & 70 \\
\hline Valencia (UV) & 42 & 30 \\
\hline Zaragoza (UNIZAR) & 268 & 86 \\
\hline Investigadores en otros centros & & 13 \\
\hline TOTAL & 2774 & 602 \\
\hline
\end{tabular}

Fuente: elaboración propia.

El periodo de recogida de respuestas se extendió desde el 28 de junio de 2016 hasta el 31 de julio de 2016. En total, se envió el CAWI a 2.774 investigadores universitarios de los 57.641 investigadores universitarios que había en España en ese momento, según los Indicadores del Sistema Español de Ciencia, Tecnología e Innovación 2015 del Observatorio ICONO (FECYT, 2015: 54). En total, se obtuvieron 602 respuestas al cuestionario, lo que indica un índice de respuesta del $\mathbf{2 1 . 7 0 \%}$. Estos datos se han almacenado de forma anonimizada por los autores del estudio. El tamaño de esta muestra permite obtener unos resultados con un nivel de confianza del $95 \%$ y con un margen de error del $3.97 \%$ Estos cálculos se han realizado utilizando la fórmula para el cálculo del error muestral en poblaciones finitas, donde: e=error muestral; $Z=$ constante que depende del intervalo de 
confianza (1.96 para un intervalo del 95\%); $\sigma=$ desviación típica de la población (estimada en 0.5$) ; n=$ tamaño muestral; y $\mathrm{N}=$ tamaño de la población.

$$
e=Z \frac{\sigma}{\sqrt{n}} \sqrt{\frac{N-n}{N-1}}
$$

El tratamiento de los datos se realizó mediante el paquete estadístico $R$ ( $R$ Development Core Team, 2013). Dada la distribución de los datos (no cumplen el principio de normalidad y no son homocedásticos) se decidió que la mejor prueba estadística que se podía aplicar era el test de Kruskal-Wallis, pues tal y como se puede observar en la literatura publicada (McCrum-Gardner, 2008; Hecke, 2012), este test se muestra como una de las pruebas estadísticas más robustas cuando se trata de comparar series de datos ordinales que no cumplen los principios de normalidad y homocedasticidad, tales como las escalas Likert, resultando por tanto en una buena alternativa no paramétrica a los tests paramétricos que no podrían aplicarse sobre este tipo de datos. La aplicación de esta prueba se basa en la distribución del Chi cuadrado y, por tanto, se utiliza para contrastar la hipótesis nula de independencia. Por tanto, cuando el test resulta significativo debemos inferir una relación de dependencia entre las variables enfrentadas. Para aplicar el test de Kruskal-Wallis se empleó el paquete estadístico $\mathrm{R}$, que incluye en el núcleo del programa la función necesaria para aplicar dicho test sobre cualquier conjunto de datos numéricos. Además, se valoraron los resultados generales de forma descriptiva como un gran avance para el área de conocimiento, pues no ha habido ninguna investigación hasta ahora que haya mostrado datos similares en España.

\section{Resultados}

En primer lugar, se realizó un análisis descriptivo de las respuestas del cuestionario, que mostraron la distribución de las respuestas por la edad, género, categoría profesional o área de conocimiento del personal investigador. Estos primeros resultados permitieron conocer las principales características del personal investigador universitario español proactivo con las actividades de comunicación de sus investigaciones (Alonso Flores, 2018:23). En relación con la edad, la mayoría de los investigadores que respondieron a la encuesta tenían entre $45-54$ años (un 36.7\%), seguidos por los comprendidos entre 35-44 años (un $28.9 \%$ ) y 55-64 años (22.4\%). Los jóvenes entre $25-34$ años representaron un $8 \%$ y los mayores de 65 años, un $4 \%$. Es decir, un $88 \%$ tenían entre 35 y 65 años. Con respecto al género, la mayoría de las respuestas provienen de hombres: un $71.4 \%$, frente a un $28.6 \%$ de mujeres. En el Sistema Universitario Español, el 39.4\% del Personal Docente Investigador en las universidades públicas son mujeres, según datos oficiales (Ministerio de Educación, Cultura y Deporte, 2015: 133), lo que indica un pequeño desajuste porcentual (de más de un diez por ciento) en cuanto a la representación según el género. En cuanto a la categoría profesional de los investigadores, el $37.2 \%$ eran profesores titulares y el $26.4 \%$ catedráticos de universidad. Estas dos figuras, personal docente investigador estable y funcionario en la escala universitaria española, suman un $63.6 \%$ de las respuestas. Sin embargo, la presencia de estas categorías en la universidad española es del 47.06\%, según las citadas fuentes oficiales.

Según las áreas de investigación, siguiendo los campos de conocimiento que utiliza la Comisión Nacional Evaluadora de la Actividad Investigadora (Ministerio de Educación y Formación Profesional, 2018), se observaron más respuestas en Ingenierías de la Comunicación, Computación y Electrónica (17.1\%), Ciencias de la Naturaleza (13\%), 
Ciencias Sociales, Políticas, del Comportamiento y de la Educación (11.8\%) y Ciencias Biomédicas (10.6\%). Por contra, las cuatro áreas menos visibles fueron: Ciencias Económicas y Empresariales (2.7\%), Filosofía, Filología y Lingüística (2\%), Derecho y Jurisprudencia (1.2\%) y Transferencia de Conocimiento e Innovación (1.2\%).

Figura 1. Importancia otorgada a las redes sociales en función del género en el caso del personal investigador.

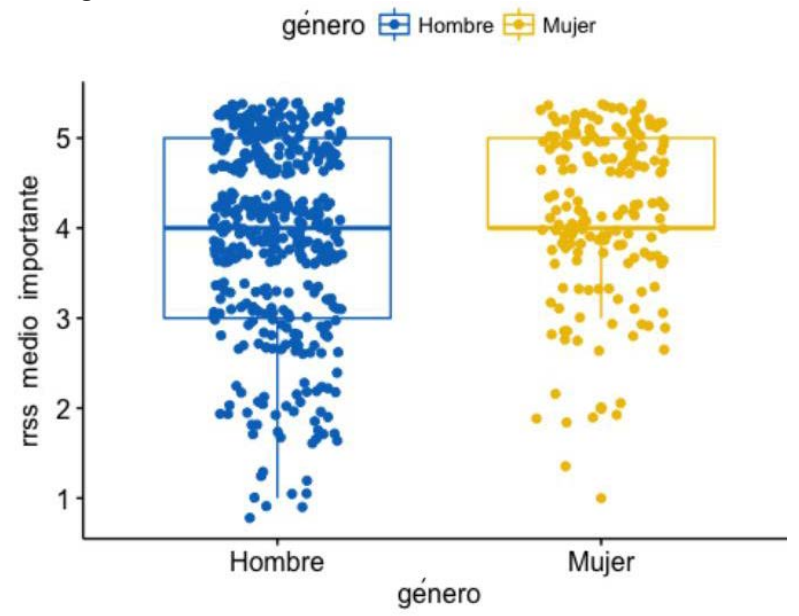

Fuente: elaboración propia. Figura basada en el género del personal investigador y su grado de acuerdo con la siguiente afirmación: "Las redes sociales son un instrumento importante para mejorar la comunicación científica".

En relación con el uso de las redes sociales, ante la pregunta de si afectan de forma positiva a la comunicación científica, tres de cada cuatro investigadores $(74.6 \%)$ estaba de acuerdo en que las redes sociales eran un instrumento importante (35.9\%) o, muy importante, (38.7\%) para mejorar la comunicación científica. Sin embargo, menos de la mitad de los investigadores (41.4\%) tenían un perfil abierto en las redes sociales que fuera activo en comunicación científica. Según el análisis estadístico, se evidencia una diferencia de género en la apreciación del personal investigador en este ámbito. En general, las investigadoras perciben las redes sociales como un medio importante más que los investigadores, tal y como se puede apreciar en los resultados representados en la figura 1. Así lo indica el test de Kruskal-Wallis, con un p-valor inferior a 0.05 , una prueba especialmente robusta en su aplicación sobre las escalas Likert (como la utilizada en esta pregunta del cuestionario). Como se puede observar, en la comparativa de los gráficos de caja y de bigotes, en el caso de las mujeres, la mayor parte de las observaciones asignan una valoración a las redes sociales de entre 4 y 5 , por lo que el diagrama es mucho más estrecho que en el caso de los hombres, cuya valoración sigue una distribución mucho más equidistante. Las redes sociales también son percibidas de manera diferente en función de la edad, según se aprecia en las respuestas. La figura 2 permite percibir que cuanto más jóvenes son los investigadores, mayor importancia le otorgan a las redes sociales. Es decir, cuanto más jóvenes son los encuestados, más agrupadas se encuentran las respuestas entre las valoraciones 4 y 5 .

Figura 2. Importancia otorgada a las redes sociales en función de la edad del personal investigador. 


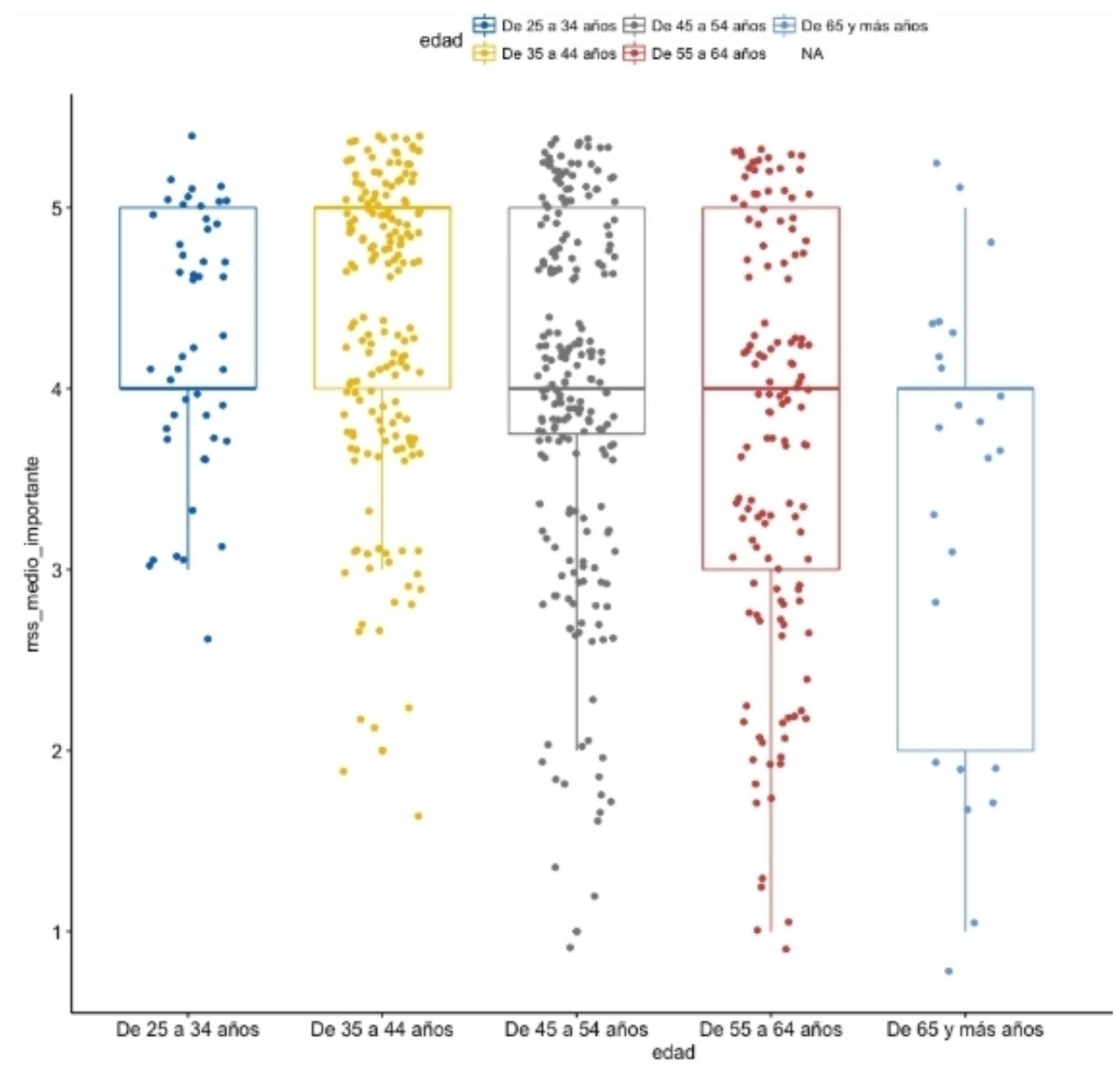

Fuente: elaboración propia. Figura basada en la edad de los investigadores y su grado de acuerdo con la siguiente afirmación: "Las redes sociales son un instrumento importante para mejorar la comunicación científica".

Según estos resultados, el tramo de edad que más importancia otorga a las redes sociales es el referente al personal investigador que oscila entre los 35 y 44 años, destacando especialmente frente a los más jóvenes ( 25 a 34 años). Este dato hay que interpretarlo con cierta cautela, pues precisamente este último es el rango de edad donde hay un menor número de observaciones, por lo que el tamaño podría estar influyendo en la distribución. En este sentido, haría falta realizar un estudio más profundo para recabar más información que permitiera corroborar esta observación, aunque los resultados obtenidos en el test de Kruskal-Wallis que relaciona las redes sociales con el número de años que se lleva investigando apunta en esta dirección, tal y como se advierte en la figura 3.

Figura 3. Importancia otorgada a las redes sociales según la experiencia investigadora de los profesores 


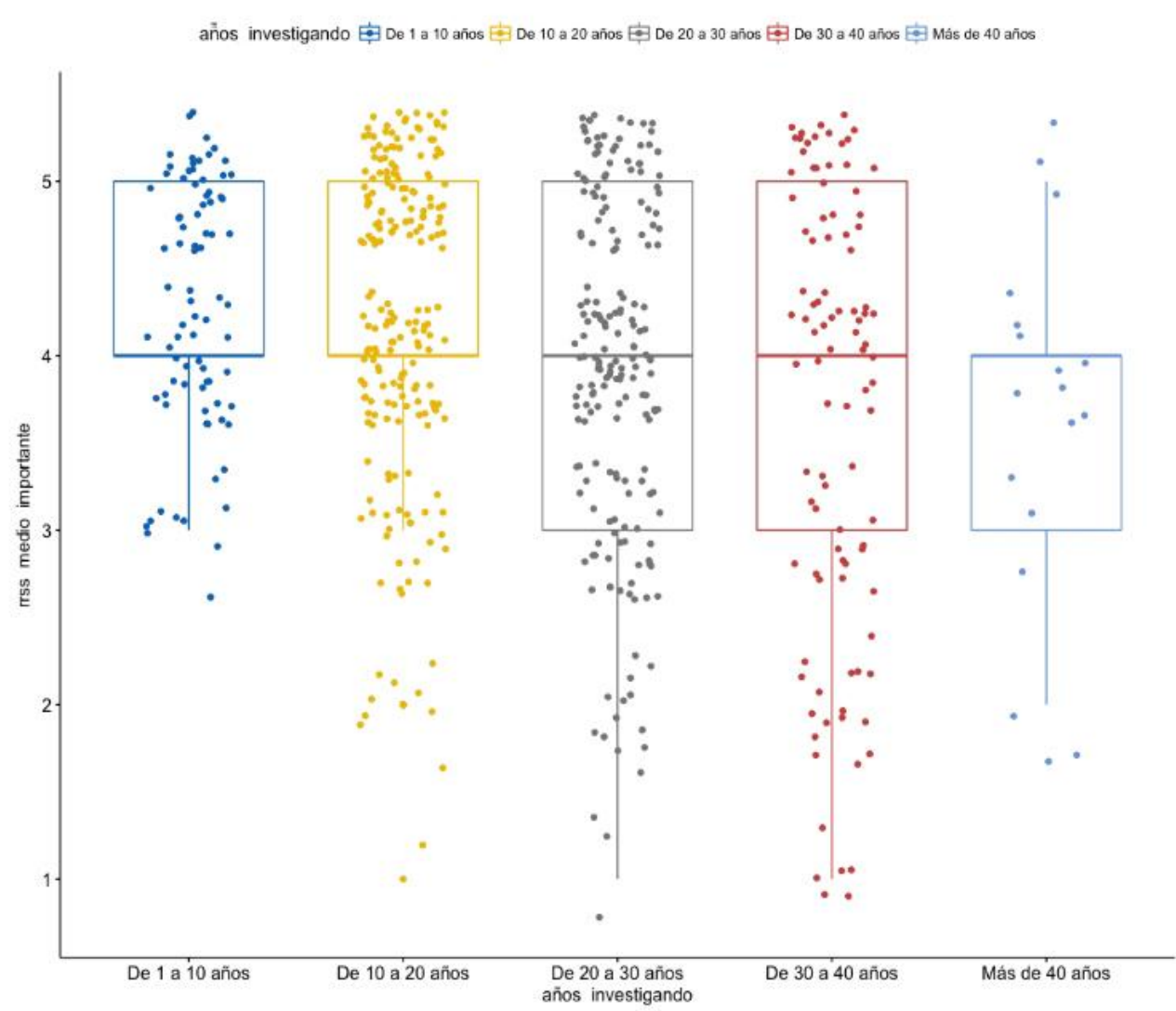

Fuente: elaboración propia. Figura basada en la experiencia de los investigadores y su grado de acuerdo con la siguiente afirmación: "Las redes sociales son un instrumento importante para mejorar la comunicación científica".

Este tipo de gráficos muestra la distribución de las variables enfrentadas, lo que permite observar visualmente las relaciones de dependencia entre los parámetros comparados (en este caso, redes sociales y género). Para su correcta interpretación, debe tenerse en cuenta la localización de los puntos a lo largo de las cajas, la distribución de las propias cajas, así como la posición de las medianas en cada caso, que nos indica el punto en el que las distribuciones son equidistantes. En los casos estadísticamente significativos debe interpretarse que existe relación de dependencia (aunque no necesariamente de causalidad) entre las variables enfrentadas.

El análisis estadístico también ha evidenciado una relación de dependencia entre el hecho de contar con redes sociales y la valoración que se hace de las mismas. En este sentido, los investigadores que cuentan con un perfil activo en redes sociales (es decir, aquellos que las utilizan habitualmente) también les otorgan más importancia que aquellos investigadores que no tienen actividad en redes sociales. Esa diferencia en la valoración puede deberse al desconocimiento de su funcionamiento, de la audiencia que alcanzan o de la percepción que son redes enfocadas al ocio, entre otros motivos. Por ello, sería necesario un estudio más profundo para explicar esta diferencia, que se puede apreciar claramente en la figura 4 . 
Figura 4. Importancia otorgada a las redes sociales entre el personal investigador que cuenta o no con perfiles activos en las mismas

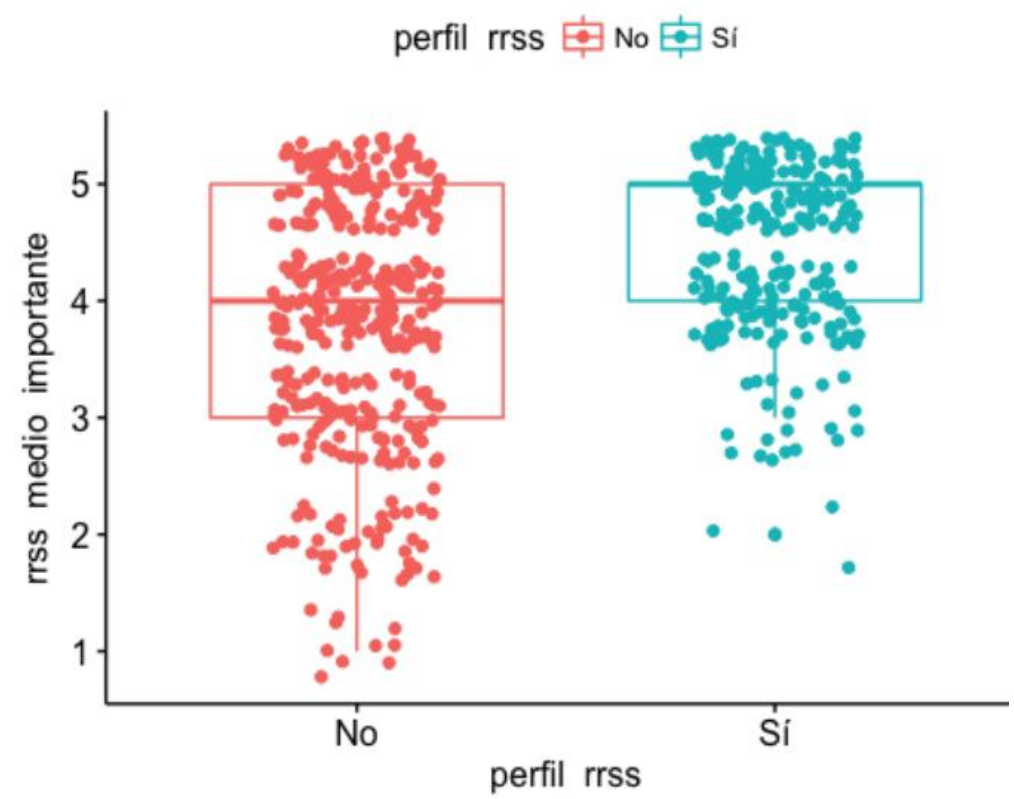

Fuente: elaboración propia. Figura que relaciona los investigadores que cuentan con redes sociales activas como Twitter y su grado de acuerdo con la afirmación: "Las redes sociales son un instrumento importante para mejorar la comunicación científica".

Por último, ante la pregunta sobre si habían percibido beneficios después de que su investigación de diera a conocer y apareciera en medios de comunicación, dos de cada tres investigadores (65.4\%) percibieron algún tipo de beneficio después de haber realizado la comunicación de sus resultados de investigación. Casi la mitad (46.5\%) adujo que gracias a ello la investigación había sido más conocida por sus colegas en España. Uno de cada cuatro investigadores (27.2\%), apuntó que le habían llamado para impartir conferencias sobre el tema a raíz de la publicación de la noticia y uno de cada cinco (20.9\%) había sido contactado desde empresas interesadas en la línea de investigación. En cuanto a los efectos negativos percibidos, una amplia mayoría de investigadores no había percibido ningún efecto negativo (84.7\%). Sin embargo, uno de cada diez encuestados aproximadamente $(8.1 \%)$, pensaba que se había tergiversado o interpretado de forma errónea la noticia en los medios de comunicación.

\section{Discusión y conclusiones}

Esta investigación se basa en una muestra autoseleccionada que consideramos que resulta lo suficientemente alta y significativa (con un nivel de confianza del $95 \%$ y margen de error del 3.97\%) como para que las conclusiones puedan ser representativas del universo al que nos referimos: el personal docente investigador de las universidades españolas que participa en actividades de divulgación científica.

En este contexto, si tenemos en cuenta estudios previos, el porcentaje de investigadores que suele participar en este tipo de acciones de divulgación científica es reducido. Según un estudio realizado en Reino Unido (The Welcome Trust, 2000: 33), el 13\% de los científicos británicos había publicado algún artículo de divulgación científica en la prensa y solo el $29 \%$ había atendido a periodistas de los medios de comunicación en el último año. 
Otra encuesta posterior (Royal Society, 2006: 10), arroja datos similares: el 20\% de los científicos e ingenieros consultados habían participado en el debate o diálogo público y el $25 \%$ de los científicos e ingenieros consultados habían escrito artículos en publicaciones no especializadas. En el caso español, algunos informes también coinciden en que la participación de los científicos en este tipo de actividades de divulgación de la ciencia sigue siendo escasa y limitada a determinados foros o medios (Martín Sempere y Rey Rocha, 2007: 53). Otro informe más reciente que analiza la participación de los investigadores del Consejo Superior de Investigaciones Científica en actividades de divulgación (OlmosPenuela, Castro-Martínez, Fernández-Esquinas, 2014) aporta más detalles, al señalar que en los últimos tres años el $54 \%$ del personal investigador de este centro español no había publicado ningún artículo en prensa o que el $65.7 \%$ no había participado en programas de radio o televisión en ese periodo.

En base a los resultados obtenidos sobre las principales características del personal investigador universitario español proactivo con las actividades de comunicación de sus investigaciones, se puede dibujar un perfil: varón, de mediana edad, funcionario público (profesores titulares) y con cierta experiencia (entre 10 y 30 años) en la investigación (Alonso Flores, 2018:26). En relación a la edad, se observa que el personal investigador que tiene entre 25 y 34 años apenas aparece representado (un 8\%), cuando se supone que es un periodo en el que se publican bastantes trabajos académicos que podrían ser adecuados para comunicarse al gran público. En este sentido, sería interesante profundizar en el análisis de esta cuestión para tratar de analizar la escasa motivación de este colectivo para participar en este tipo de acciones. Esto podría ocurrir, entre otras razones, por diversas causas complejas, como: la falta de reconocimiento oficial de la divulgación en el currículo investigador (Lázaro Real, 2015), por el esfuerzo que les requiere una actividad que causa cierta indiferencia en las instituciones (Gascoigne y Metcalfe, 1997: 265) o de cierta concepción de la divulgación como algo recreativo que solo se permite a los investigadores que se supone que ya han demostrado todo (López Cerezo, 2014), por ejemplo. El colectivo de jóvenes científicos, como los becarios predoctorales que realizan el doctorado, es de particular importancia en este sentido, según ciertos autores (Pearson, 2001: 121). En España, por ejemplo, se ha comprobado que la participación de los jóvenes científicos en actividades de divulgación como la Feria de la Ciencia de Madrid está motivada en gran medida por la satisfacción personal o el disfrute (Sempere et al, 2008: 349).

Por otra parte, la mayoría de las respuestas a la encuesta (un 63.6\%) provienen de profesores titulares (37.2\%) y catedráticos $(26.4 \%)$, cuando la presencia de estas categorías en la universidad española es del $47.06 \%$. En este sentido, algunos estudios previos han señalado que los científicos más activos en la difusión pública de la ciencia son también más activos académicamente (Jensen et al, 2008: 527) e incluso que el personal académico universitario con publicaciones divulgativas tiene niveles más altos en sus publicaciones científicas y ranking académico (Bentley y Kyvik; 2011: 48). Este último estudio encontró una correlación positiva entre publicaciones científicas y las divulgativas consistentes en todos los campos académicos del personal universitario de 13 países (Alemania, Argentina, Australia, Brasil, Canadá, Estados Unidos, Finlandia, Hong Kong, Italia, Malasia, México, Noruega y Reino Unido).

En relación con el género, predominan las respuestas de los hombres (71.4\%), mientras que en las universidades españolas representan el 6o.6\% del Personal Docente Investigador. En relación con la presencia en los medios de comunicación, un estudio reciente (Francescutti, 2018: 44) ha puesto en evidencia la infrarrepresentación del colectivo de 
investigadoras en los medios: el $23.7 \%$ de participación femenina en las fuentes expertas de la prensa es bastante inferior al $39 \%$ de científicas activas en el sistema nacional español de I+D. Es decir, las fuentes científicas masculinas citadas por la prensa de referencia triplican en número a las femeninas. Por todo ello, podría resultar interesante realizar un estudio de género con más profundidad para analizar la diferencia representativa que hemos detectado en los resultados de la encuesta.

Según las percepciones de los investigadores que han participado en esta encuesta, existen beneficiosos derivados de la participación en campañas de comunicación (Alonso Flores y Moreno Castro, 2018: 39). Después de dar a conocer su trabajo a los medios, la gran mayoría (84.7\%) no percibe ningún perjuicio, mientras que a uno de cada cuatro $(27.2 \%)$ les han solicitado que impartieran conferencias sobre el tema y uno de cada cinco (20.9\%) ha sido contactado por empresas interesadas en la línea de investigación, lo que puede mejorar las sinergias con el entorno industrial y empresarial. No obstante, para poder contrastar estas percepciones, se podría abordar otro estudio posterior más amplio y exhaustivo con la utilización de indicadores del sistema de I+D+i. El reto, en este caso, es encontrar los indicadores adecuados para conseguir evidenciar una relación de alta probabilidad causa-efecto a la hora de valorar el impacto de la comunicación social de la ciencia.

En relación con las redes sociales, este estudio se ha centrado en Twitter y no en otras redes sociales generalistas (Instagram o Facebook) u otras especializadas (como Academia.edu o ResearchGate.net), lo que supone cierta limitación parcial en cuanto a la concepción general de redes sociales que se debe exclusivamente a la selección de una muestra viable para la investigación abordada. No obstante, los resultados obtenidos arrojan algunas conclusiones interesantes. Por ejemplo, la mayoría de los investigadores (74.6\%) piensa que las redes sociales como Twitter son un instrumento útil para mejorar la comunicación científica. Sin embargo, menos de la mitad (41.4\%) dispone de ningún perfil activo en las mismas, lo que parece apuntar a que pueden tener cierta reticencia a utilizarlas. En esta línea, un estudio australiano, que analiza los beneficios e inconvenientes de utilizar las redes sociales como profesor universitario (Lupton, 2014:3), cita algunas de las preocupaciones más habituales a la hora de utilizar redes sociales, como posibles problemas de privacidad, la confusión de límites entre lo personal y lo profesional, el riesgo de poner en peligro la carrera por comentarios imprudentes, la falta de credibilidad y de calidad de los contenidos, el tiempo que hay que dedicar a las redes, la posibilidad de que se conviertan en un espacio donde recibir ataques, un exceso de autopromoción por parte de otros, el posible plagio de las ideas o problemas que puedan surgir con la comercialización de contenidos y con los derechos de autor. En nuestro caso, sería interesante analizar en un estudio posterior las razones por las que el personal investigador de las universidades muestra esta reticencia a la utilización de las redes sociales.

La prueba de Kruskal-Wallis ha permitido encontrar una relación de dependencia con la variable del género, lo que evidencia que en general las investigadoras perciben las redes sociales como un medio importante más que los investigadores. Esta posible diferencia se podría asociar al aspecto social y cooperativo de las redes sociales, relacionado con la dependencia de género en este contexto, una cuestión que se remonta a Darwin, quien afirmó que las mujeres son menos egoístas y que los hombres son más competitivos. Algunos estudios realizados en el ámbito de la Economía (Eckel y Grossman, 1998: 726) apuntan que las mujeres son más sociables (y desinteresadas) que los hombres, que serían más individualistas (y egoístas). Otros estudios en el ámbito de la cooperación entre 
sujetos (Molina et al, 2013) también encuentran una diferencia de género en el nivel de cooperación, así que sería interesante abordar de manera más profunda esta perspectiva de género en relación con la utilización de las redes sociales por parte de las investigadoras y los investigadores. Además, investigaciones más recientes, que han analizado el rol de género en la organización de estructuras sociales (Psylla et al, 2017), apuntan a la existencia de diferencias de género inherentes en el comportamiento de movilidad y en los rasgos de personalidad. La relevancia de estos estudios para la interpretación de los resultados de la muestra de la encuesta es que las investigadoras han presentado una percepción más positiva del uso cooperativo de las redes sociales.

Las redes sociales también son percibidas de manera diferente en función de la edad, según se aprecia en las respuestas de la encuesta. En general, se observa cierta tendencia a otorgar una mayor importancia a al uso de las redes sociales cuanto más jóvenes son los investigadores. Sin embargo, el tramo de edad en el que más importancia se otorga a las redes sociales es el referente al personal investigador que oscila entre los 35 y 44 años, por encima de la franja de 25 a 34 años. La horquilla de edad de los 35 a los 44 años es la que sitúa al personal de investigación en una posición más estable, laboralmente, frente a la primera franja de edad en la que todavía se suele estar en proceso de formación. Por lo que se podría inferir que los jóvenes que están estabilizados laboralmente son más proactivos al uso de las redes sociales para difundir los resultados de sus investigaciones.

Para concluir, el análisis estadístico también señala que existe una relación significativa entre el hecho de contar con redes sociales y la valoración que se hace de las mismas. En este caso, quienes cuentan con un perfil activo en redes sociales también otorgan más importancia a las mismas que aquellos investigadores que no tienen actividad en redes sociales. Esa diferencia en la valoración puede deberse al desconocimiento de su funcionamiento, de la audiencia que alcanzan o de la percepción que son redes enfocadas al ocio, entre otros motivos, algo que se podría explorar en sucesivos estudios. Sería interesante continuar enviando este cuestionario, dentro de unos años, para comparar la evolución que se ha producido en la percepción entre los investigadores universitarios de las redes sociales, como instrumento amigo para la difusión del conocimiento, o no. También para valorar si la consideración de la divulgación como un elemento requisito para la promoción en el currículo del investigador, pudiera propiciar que el uso de las redes sociales aumentara. 


\section{Apéndice 1:}

Preguntas del cuestionario, junto con las posibles respuestas, empleadas en este trabajo:

1. ¿Ha informado a través del gabinete de comunicación o de la UCC+i de alguna investigación suya publicada en una revista científica o de algún proyecto científico? Sí- No

2. ¿Podría indicar el año en que lo realizó? 2012 - 2013 - 2014 - 2015 - 2016 - NS/NC

3. ¿Tiene un perfil abierto en redes sociales (como Twitter) activo en comunicación científica? Sí - No

4. Indique su grado de acuerdo con la siguiente afirmación (en una escala de 1 a 5: 1 es totalmente en desacuerdo; 2 bastante en desacuerdo; 3 ni de acuerdo ni desacuerdo; 4 bastante de acuerdo; 5 muy de acuerdo)

"Las redes sociales son un instrumento importante para mejorar la comunicación científica"

5. ¿Ha percibido beneficios después de que su investigación se diera a conocer a través de la UCC+i y apareciera en medios de comunicación? (Marcador múltiple; seleccione al menos una y tantas opciones como quiera)

-La investigación ha sido más conocida por otros colegas investigadores en España -La investigación ha sido más conocida por colegas extranjeros - Ha aumentado el número de citas del paper después de la publicación de la noticia -Me han llamado para impartir conferencias sobre el tema a raíz de la publicación de la noticia

- Me han contactado de empresas interesadas en la línea de investigación -He firmado un artículo 83 o algún acuerdo con empresas con posterioridad a la divulgación de este trabajo de investigación.

-He encontrado algún patrocinio o mecenazgo de la línea de investigación después de la difusión pública de la noticia. -He recibido algún premio o reconocimiento después de la divulgación de la investigación -No he percibido ningún tipo de beneficio. -Otro (¿podría especificar?):

6. ¿Ha percibido perjuicios después de que su investigación se diera a conocer a través de la UCC+i y apareciera en medios de comunicación? (Marcador múltiple; seleccione al menos una y tantas opciones como quiera)

- Ha sido una pérdida de tiempo: le he dedicado esfuerzo y no percibo ningún reconocimiento

-Es una "distracción" de los objetivos científicos: investigar y publicar -He ganado reconocimiento público a expensas de perder cierto prestigio entre colegas - He recibido críticas o desconsideración por parte de colegas por divulgar al gran público -Se ha tergiversado o interpretado de forma errónea la noticia en los medios de comunicación

-No he percibido ningún tipo de perjuicio

-Otro (¿podría especificar?):

7. En resumen, ¿considera que participar en actividades de comunicación de la ciencia ha repercutido de algún modo en su carrera profesional? En una escala de 1 a 5 (1 Me ha perjudicado. 2 Ha afectado de forma negativa. 3 No ha afectado a mi carrera. 4 Ha resultado positivo. 5 Me ha beneficiado)

Datos del encuestado:

8. Género: Hombre o Mujer 
9. Edad (opcional): De 25 a 34 años; De 35 a 44 años; De 45 a 54 años; De 55 a 64 años; De 65 y más años

10. Número de años investigando: De 1 a 10; De 11 a 20; De 21 a 30; De 31 a 40; Más de 41 años

11. Área científica: Matemáticas y Física; Química; Biología Celular y Molecular; Ciencias Biomédicas; Ciencias de la Naturaleza; Ingenierías: Tecnologías Mecánicas y de la Producción; Ingenierías de la Comunicación, Computación y Electrónica; Arquitectura, Ingeniería Civil, Construcción y Urbanismo; Ciencias Sociales, Políticas, del Comportamiento y de la Educación; Ciencias Económicas y Empresariales; Derecho y Jurisprudencia; Historia, Geografía y Artes; Filosofía, Filología y Lingüística; Transferencia del Conocimiento e Innovación.

12. Universidad: (texto de respuesta corta)

13. Categoría: Catedrático; Profesor titular; Profesor contratado doctor; Profesor ayudante doctor; Profesor ayudante; Profesor visitante; Profesor asociado; Profesor colaborador; Profesor emérito; Otra categoría (especificar).

\section{Agradecimientos}

Esta investigación se inició en el transcurso del Máster en Historia de la Ciencia y Comunicación Científica de las Universidades de Valencia, Alicante y Miguel Hernández de Elche y se ha desarrollado en el marco del Grupo de investigación en Cultura CientíficaScienceflows (GICC) (Ref. UV-INV_GIUV-172923) de la Universidad de Valencia. Agradecemos las opiniones y las sugerencias de numerosos profesionales del sector de la comunicación y de la divulgación científica, como: Carlos Centeno (Universidad de Granada), Esperanza García Molina (SINC), Ana Victoria Pérez (Fundación ${ }_{3} \mathrm{CIN}$ ), Gonzalo Remiro (FECYT), Elías Sanz (Universidad Carlos III de Madrid) y Luis Zurano (Universidad Politécnica de Valencia). Además, queríamos reconocer la importancia que ha tenido la colaboración de los profesionales de los gabinetes de comunicación y/o Unidades de Cultura Científica y de Innovación de todas las universidades que han participado para que la encuesta haya podido llevarse a cabo. 


\section{Referencias bibliográficas}

ALLGAIER, J. \& DUNWOODY, S. \& BROSSARD, D. \& LO, Y. \& PETERS, H.P. (2013) a): Journalism and Social Media as Means of Observing the Contexts of Scien-ce. BioScience, 63: $284-287$.

ALLGAIER, J. \& DUNWOODY, S. \& BROSSARD, D. \& LO, Y. \& PETERS, H.P. (2013) b): Medialized Science? Neuroscientists' Reflections on Their Role as Journalistic Sources. Journalism Practice, 7: 413-429.

ALONSO FLORES, F. J. (2018): Actas del VI Congreso de Comunicación Social de la Ciencia. Volumen I: Agentes y Destinatarios de la Comunicación Social de la Ciencia. UCOPress, Editorial Universidad de Córdoba. ISBN: 9788499273822.

ALONSO-FLORES, J. \& MORENO-CASTRO, C. (2018): Does Science Communication Enhance Researcher Impact? A Survey among Scientists at Spanish Universities. Journal of Education and Social Policy, 5(2):34-44.

https://www.jespnet.com/journals/Vol_5_No_2_June_2018/5.pdf

ALONSO-FLORES, J. \& ELEAZAR SERRANO-LÓPEZ, A. \& MORENO-CASTRO, C. (2018): ¿Cómo perciben los investigadores españoles la publicación de noticias sobre los resultados de sus actividades de I+D+i? InMediaciones de la Comunicación. 13 (2).

BABBITT, C.W. (2018): Communicating science for clean technology. Clean Technologies and Environmental Policy. 20 (8): 1735-1736.

BENTLEY, P. \& KYVIK, S. (2011): Academic staff and public communication: a survey of popular science publishing across 13 countries. Public Understanding of Science, 20 (1): 4863.

BIK, H.M. \& GOLDSTEIN, M.C. (2013): An introduction to social media for scientists. PLoS Biology, 11(4): e1001535.

BROSSARD, D. \& SCHEUFELE, D.A. (2013): Science, New Media, and the Public. Science, 339 (6115): 40-41

CAMPOS-FREIRE, F. \& RÚAS-ARAUJO, J. (2016): Uso de las redes sociales digitales profesionales y científicas: el caso de las 3 universidades gallegas. El profesional de la información, 25, 3: 431-440.

CHAPMAN, J.M. \& ALGERA, D. \& DICK, M. \& HAWKING, E.E. \& LAWRENCE, M.J. \& LENNOX R.J. ET AL (2015): Being relevant: practical guidance for early career researchers interested in solving conservation problems. Global Ecology and Conservation, 4: 334-348.

CHAVES-MONTERO, A. \& GADEA-AIELLO, W. F. \& AGUADED-GÓMEZ, J. I. (2017): La comunicación política en las redes sociales durante la campaña electoral de 2015 en España: uso, efectividad y alcance. Perspectivas de la Comunicación, 10(1). http://revistas.ufro.cl/index.php/perspectivas/article/view/675 
COOKE, S.J. \& GALLAGHER, A. J. \& SOPINKA, N.M. \& NGUYEN, V.M. \& SKUBEL, R.A. \& HAMMERSCHLAG, N; BOON, S. \& YOUNG, N \& DANYLCHUK, A.J. (2017): Considerations for effective science communication. FACETS 2: 233-248. doi:10.1139/facets-2016-0055

DARLING, E.S. \& SHIFFMAN, D. \& COTE, I.M. \& DREW, J.A. (2013): The role of Twitter in the life cycle of a scientific publication. Ideas in Ecology and Evolution, 6(1): 32-43.

DENNEN, V.P. (2014): Becoming a blogger: trajectories, norms, and activities in a community of practice. Computers in Human Behavior, 36: 350-358.

DÍAZ ROSAS, F. \& CUEVAS LÓPEZ, M. (2015): Género y liderazgo en la universidad española. Un estudio sobre la brecha de género en la gestión universitaria. Archivos Analíticos de Políticas Educativas, 23 (106).

http://www.redalyc.org/pdf/2750/275041389097.pdf

DIDEGAH, F. \& MEJLGAARD, N. \& SORENSEN, M.P. (2018): Investigating the quality of interactions and public engagement around scientific papers on Twitter. Journal of Informetrics. 12 (3): 960-971.

ECKEL, C.C. \& GROSSMAN, P.J. (1998): Are Women Less Selfish Than Men?: Evidence From Dictator Experiments. The Economic Journal, 108 (448) 726-735.

EYSENBACH, G. (2011): Can Tweets Predict Citations? Metrics of Social Impact Based on Twitter and Correlation with Traditional Metrics of Scientific Impact. J Med Internet Res 2011;13(4):e123. https://www.jmir.org/2011/4/e123/

FECYT (2012): Libro Blanco de las Unidades de Cultura Científica y de la Innovación, UCC+i. https://www.fecyt.es/es/publicacion/libro-blanco-de-las-unidades-de-cultura-cientifica-yde-la-innovacion-ucci

(Fecha de consulta: 21 de diciembre de 2018)

FECYT (2015): Indicadores del Sistema Español de Ciencia, Tecnología e Innovación 2015. ICONO: Observatorio Español de I+D+i. Ministerio de Economía y Competitividad (ahora Ministerio de Ciencia, Innovación y Universidades)

http://www.idi.mineco.gob.es/stfls/MICINN/Investigacion/FICHEROS/Estadisticas_Indica dores/Indicadores_SECTI_2015.pdf

(Fecha de consulta: 25 de octubre de 2018)

FECYT (2016): UCC+i: origen y evolución (2007-2014).

http://www.fecyt.es/es/publicacion/ucci-origen-y-evolucion-2007-2014

(Fecha de consulta: 25 de octubre de 2018)

FECYT (2018): Percepción Social de la Ciencia y la Tecnología en España https://icono.fecyt.es/informes-y-publicaciones/percepcion-social-de-la-ciencia-y-latecnologia-en-espana

(Fecha de consulta: 30 de octubre de 2018)

FILIPPO, D.D. \& SERRANO LÓPEZ, A. (2018): From academia to citizenry. Study of the flow of scientific information from projects to scientific journals and social media in the field of "Energy saving". Journal of Cleaner Production. 199, 248-256. 
FRANCESCUTTI, P. (2013). Sobre los estudios observacionales y su tratamiento periodístico. Cuadernos de la Fundación Dr. Antoni Esteve, 26:39-45.

https://www.esteve.org/capitulos/6-dialogo-2-sobre-los-estudios-observacionales-y-sutratamiento-periodistico/

(Fecha de consulta: 28 de octubre de 2018)

FRANCESCUTTI, P. (2018): La visibilidad de las científicas españolas. Cuaderno 44: Fundación Dr. Antoni Esteve. Barcelona. ISBN: 978-84-947204-2-0 www.esteve.org/wp-content/uploads/2018/07/CUADERNO_ESTEVE_44_web.pdf (Fecha de consulta: 28 de octubre de 2018)

GARCÍA FERRANDO, M. \& IBAÑEZ, J. \& ALVIRA, F. (1986): El análisis de la realidad social. Métodos y técnicas de investigación. Alianza Editorial, Madrid.

GARIMELLA, K. \& XIAO, H. (2017): Media Attention to Science. Proceedings of the 26th International Conference on World Wide Web Companion: 779-780. International World Wide Web Conferences Steering Committee.

GASCOIGNE, T. \& METCALFE, J. (1997): Incentives and Impediments to Scientists Communicating Through the Media,18 (3): 265-282.

GONZÁLEZ-DÍAZ, C. \& IGLESIAS-GARCÍA, M. \& CODINA, L. (2015): Presencia de las universidades españolas en las redes sociales digitales científicas: caso de los estudios de comunicación. El profesional de la información, 24, 5: 640-647.

GONZÁLEZ-PEDRAZ, C. \& PÉREZ RODRÍGUEZ, A. V. \& CAMPOS-DOMINGUEZ, E. \& QUINTANILLA, A. \& FISAC, M. (2018): Estudio de caso sobre las Unidades de Cultura Científica (UCC+i) españolas en la prensa digital. Doxa Comunicación. Revista interdisciplinar de estudios de comunicación y ciencias sociales. 169-189.

HALL, N. (2014): The Kardashian index: a measure of discrepant social media profile for scientists. Genome Biology, 15: 424 .

HECKE, T. V. (2012): Power study of anova versus Kruskal-Wallis test. Journal of Statistics and Management Systems, 15(2-3), 241-247.

JENSEN, P. \& ROUQUIER, J.B. \& KREIMER, P. CROISSANT, Y. (2008): Scientists who engage with society perform better academically. Science and Public Policy, 35 (7): 527541.

JOHNES, J. (2018): University rankings: What do they really show? Scientometrics, 115(1): $585-606$.

KWOK, R. (2018): Press ahead. Nature. 56o (7717): 271-273.

LAMB, C.T. \& GILBERT, S.L. \& FORD, A.T. (2018): Tweet success? Scientific communication correlates with increased citations in Ecology and Conservation. 6 (e4564). 
LÁZARO REAL, E. (2015): "Las universidades españolas y la divulgación científica". Blog de la Asociación Española de Comunicación Científica.

www.aecomunicacioncientifica.org/universidades-espanolas-y-divulgacion/

(Fecha de consulta: 27 de octubre de 2018).

LIANG, X. \& SU L. Y. \& YEO, S. K. \& SCHEUFELE, D. A. \& BROSSARD, D. \& XENOS, M. \& NEALEY, P. \& CORLEY, E. A. (2014): Building Buzz: (Scientists) Communicating Science in New Media Environments. Journalism \& Mass Communication Quarterly, 91(4):772-791.

LÓPEZ CEREZO, J. A. (2014): "Existen incentivos para no divulgar la ciencia". Entrevista realizada por el departamento de Cultura Científica del CSIC. Agencia SINC (Servicio de Información y Noticias Científicas).

www.agenciasinc.es/Entrevistas/Existen-incentivos-para-no-divulgar-la-ciencia (Fecha de consulta: 27 de octubre de 2018).

LUPTON, D. (2014): 'Feeling Better Connected': Academics' Use of Social Media. Canberra: News \& Media Research Centre, University of Canberra.

https://www.canberra.edu.au/about-uc/faculties/arts-design/attachments2/pdf/n-and$\mathrm{mrc} /$ Feeling-Better-Connected-report-final.pdf

MANDAVILLI, A. (2011): Peer review: Trial by Twitter. Nature 469, 286-287. Nature. 469. 286-7.

MARTÍN SEMPERE, M. J. \& REY ROCHA, J. (2007): El papel de los científicos en la comunicación de la ciencia y la tecnología a la sociedad: actitudes, aptitudes e implicación. Grupo de Estudios de la Actividad Científica del CSIC. Madrid. http://digital.csic.es/bitstream/10261/1616/1/30_Papel.pdf

MCCRUM-GARDNER, E. (2008): Which is the correct statistical test to use? British Journal of Oral and Maxillofacial Surgery, 46(1), 38-41.

MINISTERIO DE EDUCACIÓN Y FORMACIÓN PROFESIONAL (2018): (Ministerio de Educación y Formación Profesional, 2018). Convocatoria de Sexenios de la Comisión Nacional Evaluadora de la Actividad Investigadora.

https://www.mecd.gob.es/servicios-al-ciudadano-

mecd/catalogo/general/educacion/050920/ficha.html

(Fecha de consulta: 26 de octubre de 2018)

MINISTERIO DE EDUCACIÓN, CULTURA Y DEPORTE (2015): Datos y Cifras del Sistema Universitario Español. Curso 2014-2015. Edita: Secretaría General Técnica de la Subdirección General de Documentación y Publicaciones.

https://www.mecd.gob.es/dms/mecd/servicios-al-ciudadano-

mecd/estadisticas/educacion/universitaria/datos-cifras/Datos-y-Cifras-del-SUE-Curso-

2014-2015.pdf

(Fecha de consulta: 25 de octubre de 2018)

MOLINA, J.A. \& GIMÉNEZ-NADAL, J.I. \& CUESTA, J.A. \& GRACIA-LAZARO, C. \& MORENO, Y. \& SANCHEZ, A. (2013): Gender Differences in Cooperation: Experimental Evidence on High School Students. PLoS ONE, 8 (12) e8370o.

https://journals.plos.org/plosone/article?id=10.1371/journal. pone. 0083700 
MORENO CASTRO, C. (2004): La información científico-técnica, en Fernández del Moral, Javier (Coordinador) Periodismo especializado. Madrid: Ariel: 239-262.

MORENO SARDÁ, A. \& MOLINA RODRÍGUEZ-NAVAS, P. \& CORCOY RIUS, M. (2013): La información de las administraciones públicas locales. Las webs de los ayuntamientos de Cataluña. Revista Latina de comunicación social, 68: 21-27.

http://www.revistalatinacs.org/068/paper/987_Bellaterra/RLCS_paperg87.pdf

OLVERA-LOBO, D. \& LÓPEZ-PÉREZ, L. (2014): Science Communication 2.0: The Situation of Spain through Its Public Universities and the Most Widely-Circulated Online Newspapers. Information Resources Management Journal, 27 (3), 42-58.

OLMOS-PEÑUELA, J. \& CASTRO-MARTÍNEZ, E. \& FERNÁNDEZ-ESOUINAS, M. (2014): Diferencias entre áreas científicas en las prácticas de divulgación de la investigación: un estudio empírico en el CSIC. Revista Española de Documentación Científica, 37 (2): e040

PANIAGUA ROJANO, F.J. \& GÓMEZ CALDERÓN, B.J. \& FERNÁNDEZ SANDE, M. (2012): La incorporación de los departamentos de comunicación de las universidades españolas al entorno digital. Un análisis cuantitativo. Estudios sobre el Mensaje Periodístico, 18: 691701.

PAREJO CUÉLLAR, M. (2016): Los gabinetes de comunicación de las Universidades españolas: propuesta de modelo y análisis de las salas de prensa virtuales universitarias. Tesis doctoral. Universidad de Extremadura (España). http://dehesa.unex.es/xmlui/handle/10662/4172

(Fecha de consulta: 29 de octubre de 2018).

PAREJO CUÉLLAR, M. \& MARTÍN PENA, D. \& VIVAS MORENO, A. (2017): La divulgación científica: Estructuras y prácticas en las universidades. Barcelona: Gedisa Comunicación.

PAREJO, M. \& MARTÍN-PENA, D. \& PINTO-ZÚÑIGA, R. (2016): El nuevo rol de las universidades en la comunicación científica. Actas del I Congreso Internacional Comunicación y Pensamiento. Comunicracia y desarrollo social: 523-539.

PEARSON, G. (2001): The participation of scientists in public understanding of science activities: The policy and practice of the U.K. Research Councils. Public Understanding of Science 10 (1): 121-37.

PEOPLES, B.K. \& MIDWAY, S.R. \& SACKETT, D. \& LYNCH, A. \& COONEY, P.B. (2016): Twitter predicts citation rates of ecological research. PLOS ONE, 11(11): e0166570.

PÉREZ RODRÍGUEZ, A. V. \& GONZÁLEZ PEDRÁS, C. \& ALONSO BERROCAL, J.L. (2018): Twitter como herramienta de comunicación científica en España. Principales agentes y redes de comunicación. Communication papers: media literacy and gender studies, 7(13), 95-112.

PÉREZ RODRÍGUEZ, A. V. (2016): Imagen Visible de la Ciencia en la prensa digital generalista: Actores y Procesos (España 2002-2011). Salamanca: Universidad de Salamanca. Tesis doctoral. https://gredos.usal.es/jspui/handle/10366/133355 
PÉREZ-ESPARRELLS, C. \& LÓPEZ GARCÍA, A. (2018). Los rankings de las instituciones de educación superior: una revisión del panorama internacional. Calidad en la educación, 30.

PETERS, H. P. (2013): Gap between Science and the Media Revisited: Scientists as Public Communicators. Proceedings of the National Academy of Sciences, 110: 14102-14109. www.pnas.org/content/pnas/110/Supplement_3/14102.full.pdf

PETERS, H.P. (2014): The Two Cultures: Scientists and Journalists, Not an Outdated Relationship. Métode Science Studies Journal, 4. 163-169.

https://ojs.uv.es/index.php/Metode/article/view/3043

PSYLLA, I. \& SAPIEZYNSK, P. \& MONES, E. \& LEHMANN, S. (2017): The role of gender in social network organization. PLoS ONE 12(12): e0189873.

https://journals.plos.org/plosone/article?id=10.1371/journal. pone.0189873

R DEVELOPMENT CORE TEAM (2013): A language and environment for statistical computing. R Foundation for Statistical Computing, Vienna, Austria. ISBN 3-900051-07. URL: http://www.R-project.org

RIPOLL PENADÉS, J. (2006): "Aspectos clave de la metodología CAWI", en Investigación y marketing, 91. $\mathrm{P}$ 41-48.

ROCA MARÍN, D. (2017): La divulgación científica en la universidad desde su contextualización histórica: estudio de caso y propuesta de un modelo de divulgación para la Universidad de Murcia. Murcia: Universidad de Murcia. Tesis doctoral. http://hdl.handle.net/10201/54519

(Fecha de consulta: 29 de octubre de 2018).

ROGERS, C.L. (1986): Scientists and Journalists: Reporting Science as News. New York: The Free Press: 42-54.

SEMPERE, J \& GARZÓN-GARCIA, B. \& REY ROCHA, J (2008): Scientists' Motivation to Communicate Science and Technology to the Public: Surveying Participants at the Madrid Science Fair. Public Understanding of Science. 17 (3): 349-367.

THE ROYAL SOCIETY \& RESEARCH COUNCIL UK \& WELLCOME TRUST (2006): Science communication: survey of factors affecting science communication by scientists and engineers.

https://royalsociety.org/topics-policy/publications/2006/science-communication/

(Fecha de consulta: 28 de octubre de 2018).

THE WELCOME TRUST (2000): The Role of Scientist in Public Debate. Full Report. Market \& Opinion International (MORI). Londres. ISBN 1841290343.

https://wellcome.ac.uk/sites/default/files/wtdoo3425_o.pdf

TOMÁS, M. \& GUILLAMÓN, C. (2009): Las barreras y los obstáculos en el acceso de las profesoras universitarias a los cargos de gestión académica. Revista de Educación, 350, 253-275. www.revistaeducacion.educacion.es/re350/re350_11.pdf 
WEHRMANN, C. \& VAN DER SANDEN, C. A. (2017): Universities as living labs for science communication. Journal of Science Communication (JCOM) 16 (05) C03

https://jcom.sissa.it/sites/default/files/documents/JCOM_1605_2017_Co3.pdf 